\title{
Dinâmica de crescimento e distribuição diamétrica de fragmentos de Floresta Nativa do Cerrado na Região Oeste da Bahia, Brasil
} A distribuição diamétrica é uma ferramenta para analisar a distribuição estrutural de indivíduos arbóreos cuja finalidade é demonstrar a renovação ou não de novos indivíduos no ambiente.
Assim, esta pesquisa objetivou avaliar a estrutura diamétrica de população arbórea onde predomina espécies nativas como o Caryocar Cuneatum, popularmente conhecido como Pequi. Essa
espécie nativa do Cerrado brasileiro é de grande valor socioeconômico e de difícil geminação natural e possui grande utilidade socioeconômica e cultural e é explorada em todo o Oeste da
Bahia, o que ressalta sua importância ainda mais em análises sobre regeneração de espécies nativas. A área estudada foi localizada em fragmentos do Cerrado nativo não antropizado com
fitofisionomia de Chapadão com muitos indivíduos arbóreos com altura entre 8 e $12 \mathrm{~m}$ nos municípios de Baianópolis e Cristópolis (Bahia). Para o trabalho foram coletadas as medidas de
diâmetro à altura do peito (DAP) de toda a comunidade arbórea das parcelas implementadas, através de um inventário realizado em uma área de $1 / 2$ hectare (50 x $50 \mathrm{~m}$ ) em cada munícipio.
A estrutura diamétrica foi analisada para toda a comunidade arbórea, no entanto as árvores da família Caryocar tiveram seus dados analisados entre si e em conjunto com a comunidade,
devido sua relevante importância para a região. Para a análise estatística dos dados das parcelas utilizou-se o programa Microsoft Office Excel 2010, para obtenção das seguintes variáveis:
limite inferior, limite superior, valor central, frequência observada, frequência acumulada, área basal, área transversal, área basal em m² e área basal acumulada. Tal investigação possibilitou
aferir que as árvores apresentam assimetria positiva e curtose do tipo platicúrtica, o que demonnstra o padrão típico de floresta nativa em forma de 'J' invertido ou exponencial negativo,
porém, de forma desbalanceada. Conclui-se que a população arbórea, entre ela, a espécie Caryocar Cuneatum, está se desenvolvendo dentro do ambiente inserido, mas é requerida atenção.

Palavras-chave: Diâmetro; Pequizeiro; Sustentabilidade Ambiental; Oeste da Bahia.

\section{Growth dynamics and diametric distribution of Cerrado Native Forest Fragments in Western Bahia, Brazil}

\begin{abstract}
The diametric distribution is a tool to analyze the structural distribution of arboreal individuals whose purpose is to demonstrate the renewal or not of new individuals in the environment. Thus, this search aimed to evaluate the diametric structure of the tree population where native species such as Caryocar Cuneatum, popularly known as Pequi, predominate. This native species from the Brazilian Cerrado is of great socioeconomic value and difficult to natural twinning and has great socioeconomic and cultural utility and is explored throughout Western Bahia species from the Brazilian Cerrado is of great socioeconomic value and difficult to natural twinning and has great socioeconomic and cultural utility and is explored throughout Western Bahia,
which highlights its importance even more in studies on the regeneration of native species. The workspace was located in fragments of the native Cerrado unmanned with the physiognomy of Chapadão with many arboreal individuals with height between 8 and $12 \mathrm{~m}$ in the municipalities of Baianópolis and Cristópolis-Bahia. For the study, measurements of diameter at breast height (DBH) were collected from the entire tree community of the implemented plots. Through an inventory carried out in an area of $1 / 2$ hectare $(50 \times 50 \mathrm{~m})$ in each municipality. The diametric structure was analyzed for the entire arboreal community, however the trees of the Caryocar family had their data analyzed among themselves and together with the community, due to its relevant importance for the region. The statistical analysis of the data of the plots was used the Microsoft Office Excel 2010 program, to obtain the following variables: lower limit, upper limit, central value, observed frequency, accumulated frequency, basal area, transversal area, basal área in $\mathrm{m}^{2}$ and accumulated basal area. Which made it possible to assess that the trees present positive asymmetry and kurtosis of the platicurtic type, which demonstrates the typical pattern of native forest in the form of an inverted or negative exponential 'J', but in an unbalanced way. It is concluded that the arboreal population, including the species Caryocar Cuneatum, are developing within the inserted environment, but attention is required.
\end{abstract}

Keywords: Diameter; Pequizeiro; Environmental Sustainability; West of Bahia.

Topic: Desenvolvimento, Sustentabilidade e Meio Ambiente

Reviewed anonymously in the process of blind peer.
Received: $\mathbf{1 0 / 0 8 / 2 0 2 0}$

Approved: 21/09/2020
Ranieldo Barreiras Barbosa Souza (iB)

Universidade Federal do Oeste da Bahia, Brasil http://lattes.cnpq.br/2995123274670779

http://orcid.org/0000-0001-6947-2872

ranieldosouza89@gmail.com

Francisco Rubens Feitosa Junior (iD)

Universidade Federal do Oeste da Bahia, Brasil

http://lattes.cnpq.br/1603533849627421

http://orcid.org/0000-0001-7793-2138

ranieldosouza89@gmail.com

Prudente Pereira de Almeida Neto (iD

Universidade Federal do Oeste da Bahia, Brasil

http://lattes.cnpq.br/4255612296660941

http://orcid.org/0000-0001-7480-0367

ranieldosouza89@gmail.com
Paulo Roberto Baqueiro Brandão (iD

Universidade Federal do Oeste da Bahia, Brasil

http://lattes.cnpq.br/4084176901171467

http://orcid.org/0000-0002-8342-9130

ranieldosouza89@gmail.com
Referencing this:

SOUZA, R. B. B.; FEITOSA JUNIOR, F. R.; ALMEIDA NETO, P. P.; BRANDÃO, P. R. B.. Dinâmica de Crescimento e Distribuição Diamétrica de fragmentos de Floresta Nativa do Cerrado na Região Oeste da Bahia, Brasil. Revista Ibero Americana de Ciências Ambientais, v.11, n.5, p.423-436, 2020. DOI: http://doi.org/10.6008/CBPC2179-6858.2020.005.0039 


\section{INTRODUÇÃO}

O Cerrado é o segundo maior bioma brasileiro, possui uma área de aproximadamente 2.036 .448 km², cerca de aproximadamente $24 \%$ de território nacional, que compreende às áreas territoriais do Distrito Federal e da totalidade ou parte de outros dez estados, quais sejam: Goiás, Mato Grosso, Mato Grosso do Sul, Tocantins, Maranhão, Bahia, Piauí, Minas Gerais, São Paulo e Paraná (MMA, 2009).

O Cerrado, ou savana brasileira, como é conhecido mundialmente, apresenta aproximadamente 11.627 espécies nativas de flora e as principais fitofisionomias consistem em formações florestais, savânicas e campestres (Campo sujo, Campo limpo e Campo rupestre) cuja alta biodiversidade e exploração predatória das últimas décadas faz desse bioma um dos mais ameaçados do mundo, com 132 espécies da flora ameaçadas de extinção (MMA, 2009; VACCHIANO et al., 2019; GONÇALVES et al., 2019). Essa diversidade e extensão resultam, também, das áreas de transições existentes onde os elementos florísticos se misturam é resultante de processos e dinâmicas históricas e climáticas.

Uma análise sobre o futuro do Cerrado realizado por Strassburg (2017), produziu dados preocupantes. Segundo o autor, o Cerrado brasileiro pode ter um colapso em seu ecossistema devido à exploração predatória dos seus recursos naturais, onde, nas atuais circunstâncias, é provável que, até 2050, seja registrada uma redução entre 31 e $34 \%$ desse importante bioma, acarretando na extinção de aproximadamente 480 espécies vegetais, uma extinção em massa que alterará drasticamente o funcionamento dos ecossistemas locais.

A savana mais rica do mundo em biodiversidade, o Cerrado brasileiro, nas últimas décadas tornouse um dos maiores produtores de alimentos do mundo com o suporte de tecnologias adaptadas à região (DUARTE et al., 2015). O aumento da produção de grãos no bioma Cerrado exerce uma crescente pressão sobre os ecossistemas naturais, causando impactos significativos na biodiversidade, enquanto a produção de soja cresce $4,1 \%$ ao ano, ao passo que $1,2 \%$ da vegetação típica do Cerrado é condenada ao desaparecimento no mesmo interstício temporal (WWF, 2014).

Desse modo, a agricultura de precisão vem colocando em risco a biodiversidade existente no cerrado, a exemplo das últimas décadas, onde, no Oeste da Bahia, a perda de vegetação nativa atingiu níveis recordes desde o fim dos anos 1990 (BÜHLER et al., 2019). Nessa região, somente 40\% da vegetação permaneceu estável entre 1986 e 2005 e cerca de 12\% da cobertura florestal teria desaparecido, apenas entre 2000 e 2005 (BRANNSTROM, 2009).

O Oeste da Bahia compõe a mais nova fronteira agrícola nacional, o MATOPIBA, formada pelos estados Maranhão, Tocantins, Piauí e Bahia, abrangendo 337 municípios em uma área de 73.173.485 hectares (PEREIRA et al., 2016). Essa região baiana é locus de desenvolvimento do modelo agrícola moderno e exportador vigente em diversos territórios do país, com início da expansão na década de 1980 (BRANNSTROM et al., 2008; MENKE et al., 2009; SANO et al., 2009). Um dos fatores para o desmatamento da vegetação nativa nessa região é o seu relevo plano (chapadões) e precipitação suficiente para a produção de grãos, que foi acelerado no final da década de 1990 e início do século XXI (BARROSO et al., 2019). 
Os poderes públicos permitiram a transformação agrícola do bioma Cerrado, que aconteceu através da reorientação geográfica de esforços de desenvolvimento regional para fora da Amazônia, um discurso institucional elaborado por órgãos públicos que investiram na construção de uma "vocação agrícola" para o dito bioma e na sua disponibilidade para fins produtivos (CONTINI et al., 2010), silenciando, desse modo, os seus interesses ecológicos.

A região Oeste da Bahia possui cerca de 4 milhões de hectares de espaço verde nativo, dentro de propriedades rurais e particulares destinados à formação de APP (áreas de preservação permanente) do Cerrado (EMBRAPA, 2015). Porém, ainda se faz necessário maior atenção dos órgãos públicos para preservação ambiental devido a extensa faixa territorial dessa região do território estadual. No bioma Cerrado existem diversas espécies frutíferas de cores e sabores exóticos e que são utilizadas nos saberesfazeres das populações tradicionais e locais, sendo, ainda, fonte de renda e alternativas de nutrientes (ROESLER et al., 2007; SILVA et al., 2008).

O modelo de apropriação do solo adotado pelo homem tem como característica histórica a minimização da cobertura florestal (THIBAU, 2000; MACHADO, 2008). A derrubada, queima da vegetação e as intervenções para o preparo do solo, são responsáveis pela liberação do carbono nos diferentes sistemas de uso da terra (AREVALO, 2002), o que repercute no aquecimento global e diminuição das espécies endérmicas.

Por essa razão, se faz necessária a condução de pesquisas sobre distribuição diamétrica em áreas de cerrado nativo, pois, de acordo com Austregésilo et al. (2004), para a existência de fluxo contínuo dos bens e serviços provenientes das florestas, é fundamental realizar investigações que propiciem conhecimentos para manutenção da biodiversidade, possibilitando alternativas de exploração de seus produtos de forma sustentável. De modo complementar, Coraiola et al. (2003) afirmam que a base da economia florestal deve ser fundamentada no levantamento de dados do potencial da floresta em renovar seus recursos.

Conhecer o estágio atual de desenvolvimento da floresta nativa do bioma Cerrado em questão é indispensável para a compreensão das características que ela apresenta, bem como determinar futuras intervenções e garantir a contínua renovação de recursos naturais. Essa abordagem pode ser feita através da análise estrutural da vegetação, como exemplo, por meio de estudos sobre a componente florística (SANQUETTA et al., 2007; CORAIOLA et al., 2003) e pela distribuição diamétrica das árvores.

Machado (2008), afirma que as medidas de diâmetro, analisadas isoladamente, já são capazes de determinar o estado de uma árvore. Conhecer o crescimento da floresta possibilita o melhor desenvolvimento das atividades a serem realizadas no ambiente, sejam elas para fins sociais ou ambientais. A estrutura diamétrica pode ser utilizada em qualquer região, qualquer bioma e tipos de vegetação e há vários trabalhos desenvolvidos de forma a alcançar os mais diversos objetivos.

Há várias pesquisas voltadas para o auxílio da tomada de decisões que envolvem a estrutura das florestas e tornam-se essenciais para direcionar sustentavelmente o uso desses recursos. Para conservar a flora natural é preciso entendê-la em seus aspectos comportamentais diante das adversidades naturais e antropogênicos. Numa floresta nativa, essa ação possibilita projetar e conduzir atividades de conservação 
(BATISTA et al., 2015).

Com a falta de ações de preservação do Cerrado, resultante da intensificação do uso e ocupação do território, e, além disso, sabendo-se que ainda há comunidades tradicionais e/ou rurais que dependem da natureza como recurso financeiro e alimentar e levando em consideração os impactos sofridos pelo Cerrado, há a necessidade de entender o comportamento das árvores no meio em que está inserida.

Diante disso, o objetivo desta pesquisa é analisar estatisticamente o padrão de comportamento de árvores do Cerrado nativo em um fragmento florestal onde espécies como Caryocar Cuneatun estão inseridas numa comunidade arbórea situada em duas áreas dos municípios de Baianópolis e Cristópolis (Bahia). Espera-se que o presente trabalho possa motivar futuros trabalhos de natureza conservacionista na região, que ora carece de informações sobre sua fauna e flora.

\section{MATERIAIS E MÉTODOS}

\section{Área de Pesquisa}

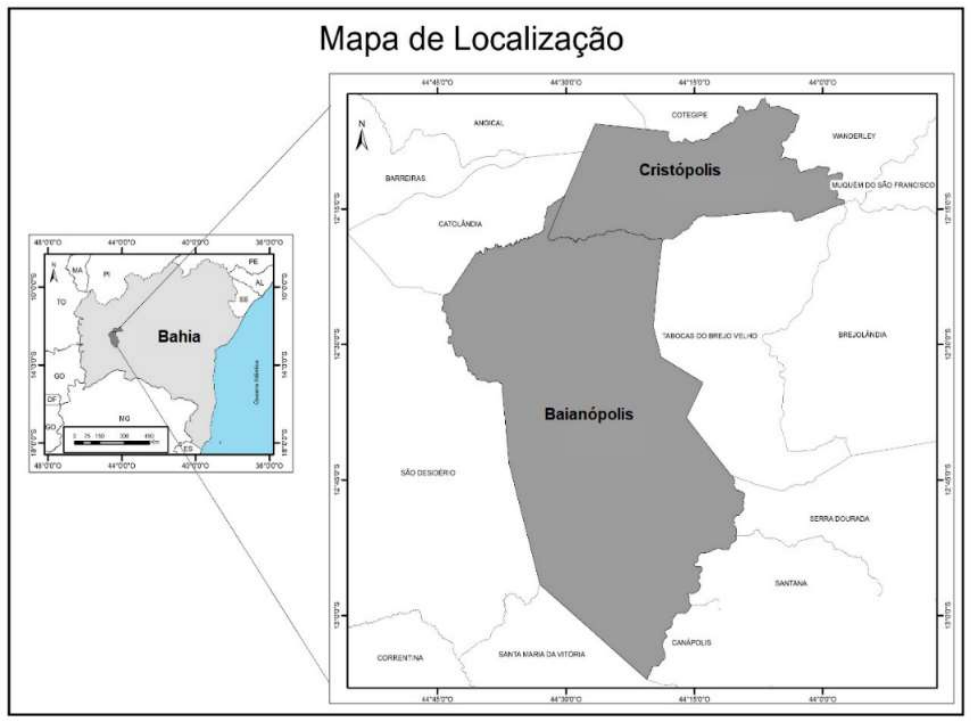

Figura 1: Localização dos municípios de Baianópolis Cristópolis no Estado da Bahia. Fonte: IBGE (2016).

O trabalho foi realizado em dois fragmentos de Cerrado dos municípios de Baianópolis e Cristópolis, unidades político-administrativas localizadas no Território de Identidade da Bacia do Rio Grande, Bahia (MDA, 2015). O município de Baianópolis possui uma extensão territorial de $3428,7 \mathrm{Km}^{2}$, com latitude de $26^{\circ} 56^{\prime} 07^{\prime \prime}$ e longitude: 44을 32' 05" W. O clima é o subúmido seco, com variações de temperaturas entre $18,8^{\circ}$ C e $25,4^{\circ} \mathrm{C}$ e precipitações pluviométricas que variam de $800 \mathrm{~mm}$ a $1.100 \mathrm{~mm}$ por ano. Em parte do município, existe uma transitoriedade para o clima semiárido, com variações médias de temperatura entre $20,7^{\circ} \mathrm{C}$ a $26,8^{\circ} \mathrm{C}$ e precipitações anuais que variam de $500 \mathrm{~mm}$ a $800 \mathrm{~mm}$ (SEI, 2011).

A área de pesquisa localizada no município de Baianópolis, situa-se no distrito de Várzea, mais precisamente na Comunidade de Brejinho. Em um levantamento feito pela CBPM-BA, a fitofisionomia vegetativa se apresenta entre arbórea do tipo Chapadão e gramíneo-lenhosa, que, por estar inserida no Bioma Cerrado é visível a sua distribuição em árvores de pequeno porte e florestas de galerias distribuídas em partes. 
A segunda área de pesquisa é no município de Cristópolis, na zona rural do município, especificamente na Fazenda Alemão. As coordenadas identificadas em latitude -1214'01"e longitude 44 는 15 . O clima é subúmido a seco e úmido, com variação de temperatura de $18,8^{\circ} \mathrm{C}$ a $25,4^{\circ} \mathrm{C}$. A extensão territorial de Cristópolis compreende uma área total de 1.052,8 km² (SEI, 2011). Ainda de acordo com a SEI (2011), a fisionomia vegetacional predominante é o Cerrado, apresentando uma fisionomia arbóreo aberto, sem floresta de galeria.

\section{Amostragem e coleta de dados}

Nas áreas investigadas, separou-se, de forma aleatória, uma área de $2.500 \mathrm{~m}^{2}$ de tamanho $50 \times 50 \mathrm{~m}$, dividida em cinco subparcelas de $10 \times 50 \mathrm{~m}$, que, ao longo da pesquisa, foi utilizada como unidade amostral permanente. Nessas áreas, foram realizados os respectivos levantamentos censitários, sendo utilizadas como amostras $100 \%$ das árvores com circunferência acima de $17 \mathrm{~cm}$, que, ao ser convertido para diâmetro, fosse possível obter um diâmetro ao nível do peito (DAP) ou altura à 1,30 m maior que $6 \mathrm{~cm}$. Foram amostrados e etiquetados todos os indivíduos arbóreos com plaquetas de alumínios numeradas sequencialmente, sendo mensurados os diâmetros ao nível de 1,30 m do solo (DAP) $>10 \mathrm{~cm}$. Para a conversão de circunferência para diâmetro $(\mathrm{cm})$ foi utiliza a fórmula:

$$
\text { D. A. } P=\frac{C}{\pi}
$$

Onde, C, é a medida da circunferência da árvore em centímetros. $\Pi$, é o número matemático de referência 3,1415.

Para as espécies arbóreas da família Caryocaraceae (pequizeiros) foram coletados folhas, galhos e flores das árvores para identificação das espécies presentes em cada local. Seguindo procedimentos citados por Ferreira (2006), após a coleta e ainda em campo, os galhos, folhas e frutos foram acondicionados sobre um jornal e, em seguida, separados uns dos outros por meio de papelão e colocados em uma prensa botânica de confecção artesanal. As flores foram acondicionas em potes de vidro esterilizados com álcool a 70\%, sendo conduzidos, em seguida, para o Herbário da Universidade Federal Oeste da Bahia (UFOB) para a devida secagem das amostras prensadas em uma estufa com temperatura aproximada de $45^{\circ} \mathrm{C}$ por tempo suficiente para completa secagem. Após esse processo, as amostras foram submetidas à identificação da espécie por meio da chave de identificação botânica de Prance et al. (1973). Foram confeccionadas exsicatas para registro e tombamento junto à coleção do herbário $B A$, sob o registro de número BRBA 8188 para Baianópolis e BRBA 8189 para Cristópolis.

\section{Distribuição de Classes Diamétricas}

Para a determinação do número de intervalos da distribuição diamétrica, da amplitude total e da amplitude de cada intervalo, foi utilizada a regra de Sturges (SANTOS et al., 2015; HIGUCHI et al., 2008):

$$
K=1+3,33 * \log N
$$

Onde: $\mathrm{K}$, é o número de intervalos a ser determinado. $\mathrm{N}$, é o número de dados a serem distribuídos. A amplitude Total é dada por: 


$$
\text { At: } \frac{d+}{d-}
$$

Onde: $\mathrm{D}+$, é o maior valor dos dados a serem distribuídos. D-, é o menor valor no conjunto de dados.

E assim, determinando a amplitude do intervalo da distribuição:

$$
A i \frac{A t}{K}
$$

Para o agrupamento e processamento dos dados obtidos, foi utilizado o software Microsoft Excel 2010.

\section{Análise estatística}

Para o primeiro momento, foram retiradas dos dados, informações que auxiliam a composição da distribuição diamétrica. São eles a média, mediana, moda, diâmetro máximo, diâmetro mínimo, amplitude, variância e desvio padrão (Tabela 1 e 2). Esses dados foram agrupados em histogramas de frequências para espécies arbóreas obedecendo a ordem da espécie de maior valor de importância (Caryocar Cuneatun), seguido da comunidade que compõe o povoamento.

Tabela 1: Parâmetros fitossociológicos de espécies arbóreas em um fragmento do Cerrado em Baianópolis (BA), por ordem crescente de (VI) - Valor de Importância (\%). Em que: (DR) - Densidade Relativa - (\%), (FR) - Frequência Relativa (\%), (DoA) - Dominância Absoluta (m/h) - (DoR) - Dominância Relativa - (\%), (VC) - Valor de Cobertura - (\%).

\begin{tabular}{|l|l|l|l|l|l|l|}
\hline ESPÉCIE & DA & DR & FR & DoA & DoR & VC \\
\hline Caryocar cuneatum & 36 & 3,86 & 100 & 16,4932 & 11,45 & 15,31 \\
\hline Demais espécies & 896 & 96,14 & 100 & 127,5429 & 88,54 & 223,6829 \\
\hline Total & 932 & 100 & 200 & 144,0361 & 100 & 238,9929 \\
\hline
\end{tabular}

Tabela 2: Parâmetros fitossociológicos de espécies arbóreas em um fragmento do Cerrado em Cristópolis (BA), por ordem crescente de (VI) - Valor de Importância (\%). Em que: (DR) - Densidade Relativa - (\%), (FR) - Frequência Relativa (\%), (DoA) - Dominância Absoluta (m/h) - (DoR) - Dominância Relativa - (\%), (VC) - Valor de Cobertura - (\%).

\begin{tabular}{|l|l|l|l|l|l|l|l|}
\hline ESPÉCIE & DA & DR & FR & DoA & DoR & VC & VI \\
\hline Caryocar cuneatum & 72 & 8,33 & 100 & 22,5046 & 59,4011 & 67,73 & 167,7311 \\
\hline Demais espécies & 792 & 91,67 & 100 & 15,3811 & 40,5988 & 132,25 & 232,2688 \\
\hline Total & 864 & 100 & 200 & 37,8857 & 100 & 200 & 399,9999 \\
\hline
\end{tabular}

Para o segundo momento foi feita a análise estatística dos dados do fragmento florestal como um todo, utilizando o programa Microsoft Office Excel 2010, obtendo-se as seguintes variáveis: limite inferior (LI), limite superior (LS), valor central (VC), frequência observada (Fo), frequência acumulada (Fa), área basal (Fo.VC), área transversal (gi), área basal (Fo.gi) e área basal acumulada (Fo.VC).

A distribuição de frequência foi observada quanto ao quociente de Liocourt 'q' que se dá pela divisão do número de indivíduos de uma classe diamétrica pelo número de indivíduos da classe anterior. Este quociente permite estimar se a comunidade vegetal se encontra balanceada e isso ocorre quando há uma razão relativamente constante de ' $q$ ' entre as classes de diâmetro (FELFILI, 1997; SCHNEIDER et al., 2000).

\section{Classificação Assimétrica e de Curtose}

Com as definições de média, moda e medianas, foi possível a determinação da assimetria ou o grau do desvio em relação ao valor central da distribuição de frequência. Na aplicação, esse parâmetro pode ser classificado como assimétrico, simétrico e negativo (ORELLANA et al., 2014). Para a definição do grau de 
elevação ou achatamento, também chamado de curtose, será utilizada a classificação de acordo com o valor de seu coeficiente em leptocúrtica $(<0,263)$, platicúrtica $(>0,263)$ e mesocúrtica $(=0,263)$, aplicando-o conforme Machado et al. (2006).

\section{RESULTADOS E DISCUSSÃO}

Foram inventariados 448 indivíduos arbóreos, sendo 421 classificados neste trabalho como espécies não identificadas e 27 indivíduos identificados, sendo da família Caryocaraceae, do gênero Caryocar e da espécie Caryocar Cuneatun Wittm (PRANCE et al., 1973). Estão distribuídos em áreas de preservação fragmentada de floresta nativa de Cerrado, no Estado na Bahia.

As estruturas das florestas nas áreas estudadas, em valor de diâmetro, seguiram padrões iguais, sendo a característica principal a presença maior de indivíduos de pequeno porte nas menores classes diamétricas, podendo-se afirmar que há um equilíbrio no balanceamento dessas distribuições. Isso se dá devido à disposição de regeneração das espécies nativas do Cerrado. Para Santos et al. (2015), ter ideia do comportamento da distribuição diamétrica de uma floresta é conhecer o seu potencial, seja sustentável ou econômico da área. Esse conhecimento é capaz de justificar a necessidade ou não de intervenções ambientais nessas localidades.

Como ordem de grau de importância (IVI), em ambas as áreas estudadas, as espécies da comunidade arbórea (não identificadas), apresentaram valores elevados, sendo 284,68 para Baianópolis e 232,26 para Cristópolis. Mas, para o interesse deste trabalho, foi adotado o Caryocar Cuneatum como espécie principal, sendo, assim, a única identificada nos fragmentos estudados. Em números de IVI temos 115,31 em Baianópolis e 167,73 em Cristópolis (ver tabelas 1 e 2). Isso significa que há uma predominância da espécie nas áreas inventariadas. Para Pereira et al. (2011), o IVI demonstra a importância da espécie no local e a sua essencialidade no equilíbrio florestal para a fauna e flora ali existentes.

Em uma pesquisa realizada por Santos et al. (2014) sobre o grau de importância das espécies do Cerrado em um munícipio de Goiás, apresentou o gênero Caryocar como das mais importantes pelo seu alto valor socioeconômico para a população da localidade. Nas áreas de realização desta pesquisa, o pequizeiro possui também alto valor social servindo como fonte de renda e alimentação para os moradores locais. Por isso a importância da espécie tratada nessa pesquisa.

Para a estatística descritiva, o diâmetro mínimo igual a 5,41 cm e o diâmetro máximo de 76,43 cm no fragmento de Baianópolis e o diâmetro mínimo de $5,28 \mathrm{~cm}$ e o diâmetro máximo de $76,75 \mathrm{~cm}$ no fragmento de Cristópolis apresentam a heterogeneidade das florestas em seus diferentes níveis de desenvolvimento. O Caryocar Cuneatum, apresenta os maiores valores de diâmetro dentro do inventário, sendo positivo o ponto de desenvolvimento do mesmo. Ecologicamente, a árvore do Caryocar apresenta grande porte arbóreo, podendo chegar a $12 \mathrm{~m}$ de altura e variando de acordo com as variações de respostas do ambiente (OLIVEIRA et al., 2010). Não há trabalhos que comprovem uma relação entre a altura da árvore do Caryocar com o seu diâmetro, podendo haver árvores pequenas com diâmetro elevado e árvores grandes com diâmetro baixo. 
Embora os diâmetros sejam considerados altos, o Caryocar apresentou baixa dispersão dos dados, se relacionado com a média nos dois municípios estudados. Essa informação é afirmada pelo Coeficiente de Variação (CV) de 69,47\% em Baianópolis e 37,23\% em Cristópolis, o que difere dos valores apresentados pelas outras árvores da comunidade com CV de 86,96\% em Baianópolis e 83,96\% em Cristópolis. Esses valores implicam afirmar que as árvores da comunidade apresentaram uma maior heterogeneidade na distribuição dos dados coletados. Os CV da comunidade nos dois municípios mostram valores muito próximos (tabelas 3 e 4). Para a Caryocar Cuneatun, há uma diferença considerável dessa variável se comparada uma área com a outra. Isso se dá, possivelmente, pela diferença na quantidade de árvores da espécie inventariada, onde a diferença em número de árvores do pequizeiro chega a 50\%. Quando a média, a mediana e a moda aparecem em pontos diferentes na distribuição o seu deslocamento pode acontecer para a direita ou para a esquerda. Esse deslocamento pode ser visto melhor na figura 2.
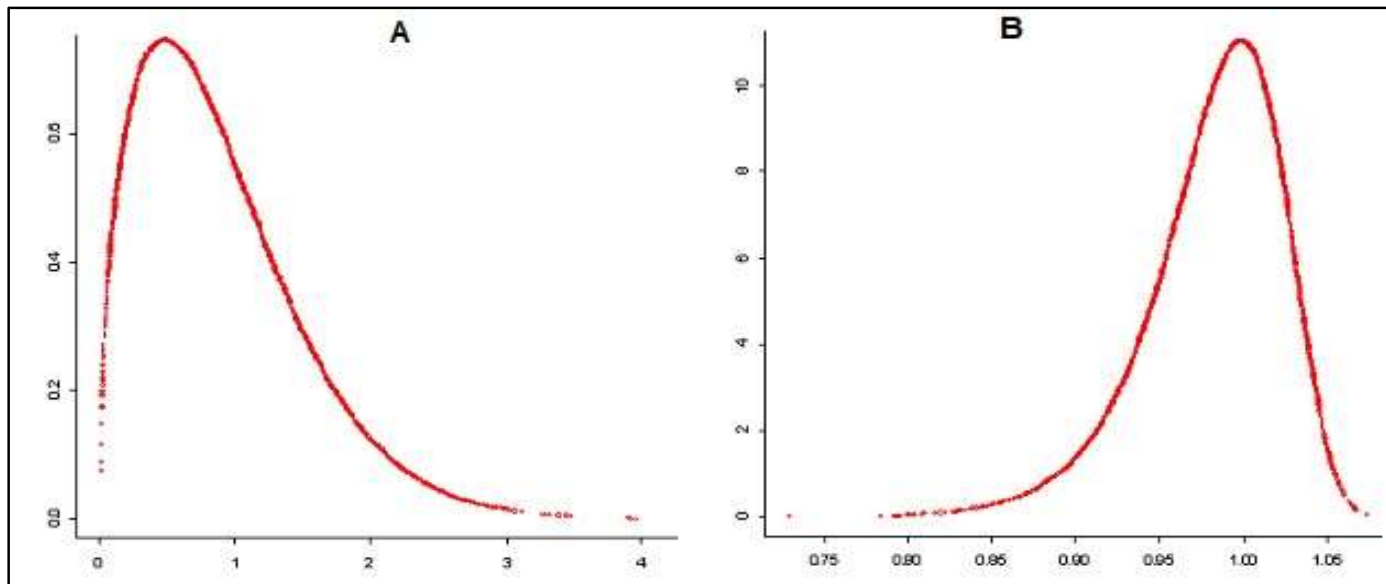

Figura 2: Exemplo de forma de distribuição com assimetria (A) e assimetria Negativa (B). Fonte: Adaptado de Machado et al. (2006)

Com isso, as curvas geradas da distribuição de diâmetros deste trabalho foram assimétricas negativa para a distribuição no fragmento de Baianópolis. Geralmente, essa assimetria se dá pelo fato de a média apresentar valor menor que o da mediana. Para a distribuição do fragmento de Cristópolis, foram assimétricas positiva, apresentando valor de média maior que o valor da mediana.

Tabela 3: Estatísticas descritivas da variável diâmetro para a comunidade arbórea e para as espécies de maior (IVI) em fragmentos do Cerrado em Baianópolis/BA.

\begin{tabular}{|l|l|l|}
\hline Medidas & Comunidade & Caryocar Cuneatum \\
\hline Média & 13,96 & 29,22 \\
\hline Mediana & 14,01 & 48,88 \\
\hline Moda & 7,01 & 7,96 \\
\hline Dmin & 5,41 & 7,96 \\
\hline Dmax & 76,43 & 72,61 \\
\hline Amplitude & 71,02 & 64,65 \\
\hline$S^{2}$ & 147,54 & 412,46 \\
\hline S & 12,14 & 20,30 \\
\hline CV\% & 86,96 & 69,47 \\
\hline Assimetria & 2,80 & 0,73 \\
\hline Curtose & 8,57 & $-0,77$ \\
\hline
\end{tabular}


Tabela 4: Estatísticas descritivas da variável diâmetro para a comunidade arbórea e para as espécies de maior (IVI) em fragmentos do Cerrado em Cristópolis/BA.

\begin{tabular}{|l|l|l|}
\hline Medidas & Comunidade & Caryocar Cuneatum \\
\hline Média & 18,39 & 40,37 \\
\hline Mediana & 7,00 & 37,89 \\
\hline Moda & 5,41 & 45,54 \\
\hline Dmin & 5,28 & 17,51 \\
\hline Dmax & 76,75 & 64,96 \\
\hline Amplitude & 71,47 & 47,45 \\
\hline$S^{2}$ & 238,44 & 226,64 \\
\hline S & 15,44 & 15,03 \\
\hline CV\% & 83,96 & 37,23 \\
\hline Assimetria & 2,21 & 0,49 \\
\hline Curtose & 2,82 & $-1,01$ \\
\hline
\end{tabular}

Na análise do grau de assimetria do coeficiente de Pearson (SPIEGEL, 2006), as comunidades arbóreas das duas áreas estudadas possuem assimetria intensa. Isso se dá pelos seus valores de coeficientes serem superiores a um (1), a saber, 2,8 em Baianópolis e 2,21 em Cristópolis. A espécie Caryocar Cuneatun apresentou grau de assimetria de 0,73 em Baianópolis e 0,49 em Cristópolis, tendo, assim, uma assimetria próxima de moderada (SCNNEIDER et al., 2009). Isso pode ser explicado devido a diferença entre moda e mediana serem menores que o valor do desvio padrão, ou seja, os valores de média e mediana são ligeiramente próximos.

Para a variável curtose (tabelas 3 e 4), as árvores das comunidades arbóreas dos dois fragmentos estudados demonstraram distribuição platicúrtica, ou seja, o achatamento da curva em seu topo teve valor positivo com relação à curva de distribuição normal ( $C=8,57$ para Baianópolis e $C=2,21$ para Cristópolis). Já o Caryocar Cuneatun, apresentou uma distribuição leptocúrtica, com curva alongada em relação à normal (C $=-0,77$ para Baianópolis e $C=-1,01$ para Cristópolis). Isso deixa claro que há uma dispersão elevada na distribuição de frequência de classes diamétricas se comparada com a dispersão de uma distribuição normal (MACHADO et al., 2006).

Devido as áreas aqui analisadas serem fragmentos de floresta nativa, as espécies arbóreas possuem particularidades ecológicas de fatores bióticos e/ou abióticos que influenciam seu comportamento no ambiente onde estão inseridos. Com isso, é esperado grande dispersão na distribuição diamétrica, fazendo com que essa distribuição não siga uma tendência de distribuição normal. Essas espécies arbóreas tendem a formar perfil de estruturas de diâmetro em forma de 'J' invertido.

Para uma tomada de decisões envolvendo o uso da estatística (seja ela descritiva, probabilística ou inferencial) de âmbito socioambiental ou econômico de sustentabilidade, a utilização dos parâmetros de assimetria e curtose se apresentam como um instrumento de mensuração da dispersão do diâmetro, fornecendo, neste caso, o grau de achatamento dessa elevação.

Considerando os fragmentos de cada área, em sua totalidade de espécies, Baianópolis apresentou média de 15,17, DAP mínimo de 5,41cm, DAP máximo de 76,46 cm, com variância de 312,41, desvio padrão de 17,67 e coeficiente de variação de 116,50. Cristópolis, por sua vez, apresentou média de 19,24, DAP mínimo de 5,28cm, DAP máximo de 76,75 cm, com variância de 255,92, desvio padrão de 15,99 e coeficiente de variação de 83,12 . Essas variáveis não diferem muito de uma área para a outra, possuindo valores bem 
aproximados. E, se isolar apenas os valores dessas variáveis para a espécie Caryocar Cuneatum, também não haverá diferença grande.

Porém, se comparado as florestas plantadas, o valor dessas variáveis, em via de regra, será sempre menor que a de floresta plantada (NETTO, 2008; CUNHA et al., 2013). Isso se dá pelo fato de se tratar de uma floresta nativa ser multiânea, ou seja, não possuir uniformidade entre os indivíduos arbóreos, sendo uma diversidade de espécies, de diferentes idades que não podem ser mensuradas e ainda estão sobre influência de fatores bióticos e abióticos.

Tabela 5: Agrupamento dos diâmetros de um fragmento de floresta nativa de Baianópolis/BA.

\begin{tabular}{|l|l|l|l|l|l|l|l|l|l|}
\hline Classe & LI & LS & VC & FO & FA & Fo.VC & Gi & Fo.Gi & Fo.VC $^{\mathbf{2}}$ \\
\hline $\mathbf{1}$ & 5,41 & 10,83 & 7,32 & 117 & 117 & 856,44 & 0,004208 & 0,492377 & 6269,141 \\
\hline $\mathbf{2}$ & 11,15 & 16,88 & 13,06 & 47 & 164 & 613,82 & 0,013396 & 0,629614 & 8016,489 \\
\hline $\mathbf{3}$ & 17,52 & 22,29 & 20,54 & 18 & 182 & 369,72 & 0,033135 & 0,596435 & 7594,049 \\
\hline $\mathbf{4}$ & 23,89 & 28,98 & 26,11 & 11 & 193 & 287,21 & 0,053543 & 0,588974 & 7499,188 \\
\hline $\mathbf{5}$ & 29,94 & 31,21 & 30,25 & 3 & 196 & 90,75 & 0,071869 & 0,215607 & 2745,188 \\
\hline $\mathbf{6}$ & 36,94 & 39,81 & 37,58 & 3 & 199 & 112,74 & 0,110918 & 0,332755 & 4236,769 \\
\hline $\mathbf{7}$ & 41,40 & 46,82 & 44,90 & 4 & 203 & 179,60 & 0,158337 & 0,633348 & 8064,04 \\
\hline $\mathbf{8}$ & 47,13 & 52,23 & 48,09 & 4 & 207 & 192,36 & 0,181635 & 0,72654 & 9250,592 \\
\hline $\mathbf{9}$ & 57,32 & 57,32 & 57,32 & 1 & 208 & 57,32 & 0,258049 & 0,258049 & 3285,582 \\
\hline $\mathbf{1 0}$ & 61,46 & 64,65 & 64,01 & 3 & 211 & 192,03 & 0,3218 & 0,965399 & 12291,84 \\
\hline $\mathbf{1 1}$ & 65,29 & 66,24 & 65,75 & 2 & 213 & 131,50 & 0,339533 & 0,679065 & 8646,125 \\
\hline $\mathbf{1 2}$ & 72,61 & 76,43 & 95,80 & 2 & 215 & 191,60 & 0,72081 & 1,44162 & 1835528 \\
\hline
\end{tabular}

LI = Limite inferior; $\mathrm{LS}=$ Limite superior; VC = Valor Central; FO = Frequência observada; FA = Frequência acumulada; Fo.VC = Área basal; Gi =área transversal; fo.gi = área basal em $\mathrm{m}^{2}$; fo.VC $\mathrm{VC}^{2}$ área basal acumulada. Fonte: Adaptada de Lima et al. (2013).

Tabela 6: Agrupamento dos diâmetros de um fragmento de floresta nativa de Cristópolis/BA.

\begin{tabular}{|l|l|l|l|l|l|l|l|l|l|}
\hline Classe & LI & LS & VC & FO & FA & Fo.VC & Gi & Fo.Gi & Fo.VC $^{2}$ \\
\hline 1 & 5,28 & 10,82 & 6,68 & 106 & 106 & 708,08 & 0,0035 & 0,3715 & 4729,97 \\
\hline 2 & 11,14 & 15,60 & 12,42 & 29 & 135 & 360,18 & 0,0121 & 0,3513 & 4473,44 \\
\hline 3 & 17,51 & 22,92 & 20,38 & 20 & 155 & 407,60 & 0,0326 & 0,6524 & 8306,89 \\
\hline 4 & 23,24 & 28,98 & 26,11 & 23 & 178 & 600,53 & 0,0535 & 1,2315 & 15679,80 \\
\hline 5 & 29,34 & 33,43 & 30,89 & 15 & 193 & 463,35 & 0,0749 & 1,1241 & 14312,90 \\
\hline 6 & 35,03 & 40,76 & 37,89 & 20 & 213 & 757,80 & 0,1128 & 2,2551 & 28713,0 \\
\hline 7 & 41,08 & 46,46 & 43,40 & 8 & 221 & 347,20 & 0,1479 & 1,1835 & 15068,50 \\
\hline 8 & 52,22 & 52,54 & 52,38 & 2 & 223 & 104,76 & 0,2155 & 0,4310 & 5487,33 \\
\hline 9 & 56,68 & 56,68 & 56,68 & 1 & 224 & 56,68 & 0,2523 & 0,2523 & 3212,62 \\
\hline 10 & 59,87 & 64,96 & 62,41 & 2 & 226 & 124,82 & 0,3059 & 0,6118 & 7790,02 \\
\hline 11 & 65,28 & 69,74 & 68,47 & 3 & 229 & 205,41 & 0,3682 & 1,1046 & 14064,40 \\
\hline 12 & 75,15 & 76,75 & 75,63 & 4 & 233 & 302,52 & 0,4492 & 1,7970 & 22879,60 \\
\hline
\end{tabular}

LI = Limite inferior; $\mathrm{LS}=$ Limite superior; VC = Valor Central; FO = Frequência observada; FA = Frequência acumulada; Fo.VC = Área basal; $\mathrm{Gi}$ = área transversal; fo.gi = área basal em $\mathrm{m}^{2} ;$ fo. $\mathrm{vc}^{2}=$ área basal acumulada. Fonte: Adaptada de Lima et al. (2013).

Pelo método estatístico (tabelas 5 e 6), é possível agrupar os dados em classes para uma melhor observação. Uma floresta nativa tem a tendência, via de regra, de apresentar uma distribuição diamétrica decrescente, em forma de ' $\mathrm{J}$ ' invertido ou exponencial negativo, que é caracterizado pela maior quantidade de indivíduos nas menores classes. Araújo et al. (2006) chamam a atenção para essa distribuição diamétrica decrescente ao afirmar que o aumento de indivíduos nas classes menores, além de significar que há uma regeneração das espécies naquele ambiente, também pode significar a perda ou morte das árvores mais velhas. Verificando o Gráfico 1, para o fragmento de Baianópolis, a classe de valor central 7,32 cm é representado por $54,42 \%$ do total, que corresponde a maior parte dos indivíduos (117). Para o fragmento de 
Cristópolis (Gráfico 2), a classe de maior representatividade de indivíduos é a 6,68, que representa 45,5\% do total (106).

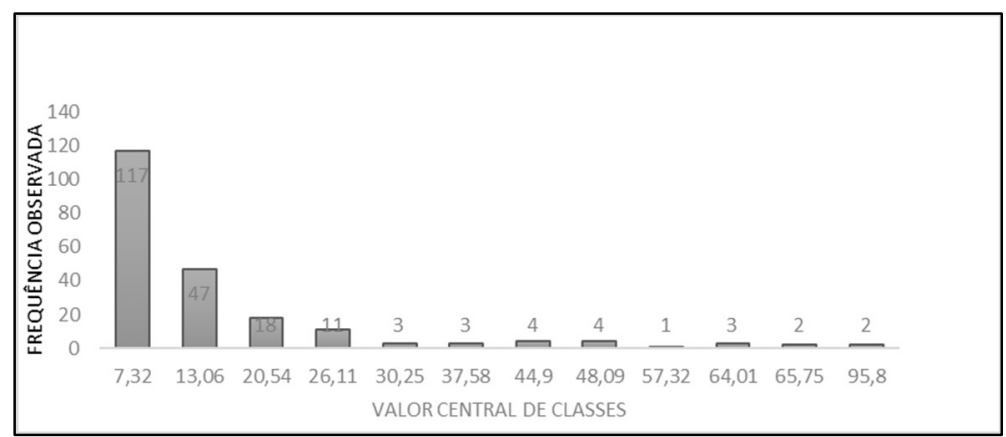

Gráfico 1: Gráfico da distribuição por classe diamétrica de um fragmento de floresta nativa do Cerrado de Baianópolis/BA.

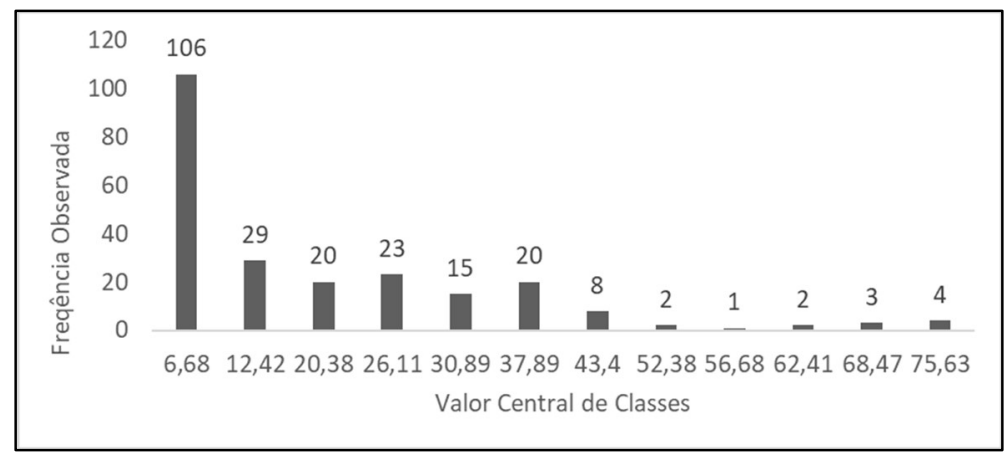

Gráfico 2: Gráfico da distribuição por classe diamétrica da população de um fragmento de floresta nativa do Cerrado de Cristópolis/BA.

A maior área basal, que determina a densidade populacional das espécies arbóreas, encontra-se na classe de diâmetro maior para o fragmento de Cristópolis $\left(1,72 \mathrm{~m}^{2} / \mathrm{ha}\right)$ e na classe intermediária de valor central 37,89 cm em Baianópolis (2,25 $\mathrm{m}^{2} / \mathrm{ha}$ ). Pelo Coeficiente de Lincoln, a presença considerável em números de árvores na menor classe diamétrica, em comparação com o número de indivíduos nas maiores classes, mostra uma distribuição desbalanceada dos indivíduos. Ao mesmo tempo em que isso sinaliza alguma taxa de mortalidade das árvores em algum momento do seu desenvolvimento, que poderia ter sido causado por vários fatores naturais e de antropização, pode indicar também que a comunidade tem potencial elevado e constante de rápida regeneração natural.

Apesar de esse tipo de distribuição diamétrica ser prevista para florestas nativas (SANTANA, 2009), para Nunes et al. (2003), a grande quantidade de indivíduos na menor classe de diâmetro pode evidenciar pressões sofridas recentemente, como o corte, as queimadas, pragas e insetos, entre outros fatores. Isso significa que o ciclo de vida das árvores não está sendo completado, havendo, assim, uma interrupção e/ou baixa brusca na escalada diamétrica, conforme sinalizado por Paula et al. (2005).

Apesar da pouca quantidade de indivíduos detectados em ambas as parcelas em relação a toda a população, os dados isolados da espécie de pequizeiro, o Caryocar Cuneatum, mostram seguir também uma tendência de distribuição diamétrica no formato de ' $J$ ' Invertido, o que assegura que o comportamento dessa espécie dentro da comunidade está positivo, se desenvolvendo como as demais espécies (Gráficos 3 e 4). 


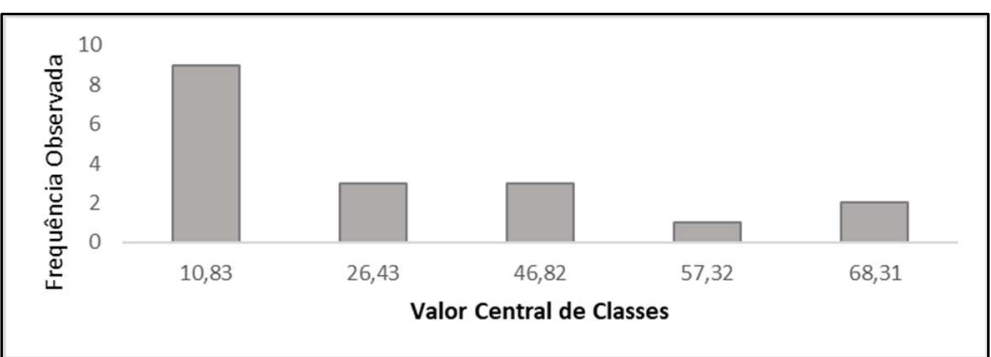

Gráfico 3: Gráfico da distribuição por classe diamétrica da espécie Caryocar Cuneatun no fragmento de floresta nativa do Cerrado de Baianópolis/BA.

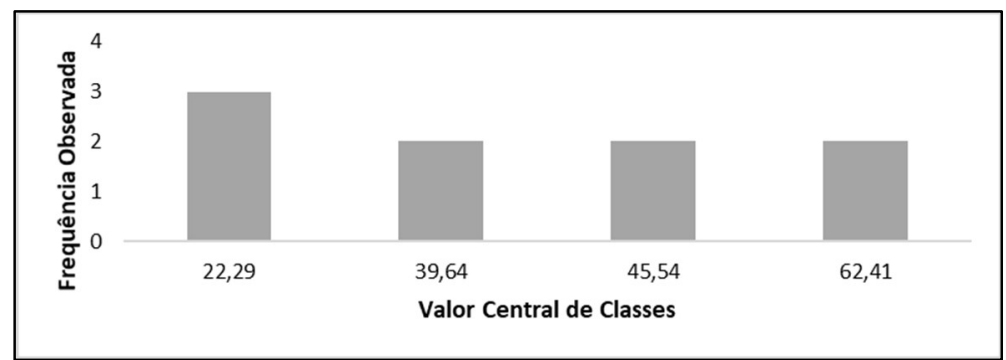

Gráfico 4: Gráfico da distribuição por classe diamétrica da espécie Caryocar Cuneatun no fragmento de floresta nativa do Cerrado de Cristópolis/BA.

Para Souza et al. (1994), conhecer a distribuição diamétrica de uma floresta, além de permitir o conhecimento e o desenvolvimento ecofisiológico de cada espécie ali presente, é um diagnosticador do estado florestal. Com isso, é possível saber o nível de exploração e a possibilidade de manejo sustentável da mesma, visando sua preservação geral ou de uma espécie específica.

\section{CONCLUSÕES}

Com base nos valores de DAP, área basal da população arbórea estudada, incluindo o Caryocar Cuneatun, fica evidente por meio da variedade do valor diamétrico, dentre outros parâmetros que os fragmentos analisados se tratam de vegetação em estágio positivo de sucessão. Os dois fragmentos de Cerrado estão em processo de desenvolvimento no que se refere ao estágio de sucessão, pois em todos os fragmentos estudados tanto a população quanto a espécie de pequizeiros apresentaram árvores pequenas em pleno crescimento. Isso indica que há um processo de regeneração natural, uma vez que há árvores em crescimento, resultando em uma possível continuidade das espécies ao longo dos anos.

Nos fragmentos de Cerrado de Baianópolis e Cristópolis o Caryocar Cuneatum, tão importante para a população local por ser fonte de renda seguem a mesma tendência de desenvolvimento, podendo se dizer que a árvore está conseguindo se regenerar em ambas as áreas estudadas. Vale observar que toda população analisada seguiu a tendência de distribuição diamétrica comum para a maioria dos estudos em florestas nativas, ou seja, frequência maior de indivíduos nas menores classes de diâmetro.

Embora a estrutura em formato de ' $\mathrm{J}$ ' tenha sido satisfatória para esse trabalho, o fato de haver poucos indivíduos em classes diamétricas intermediárias, mostra que há um desbalanceamento estrutural da floresta, indicando uma mortalidade de árvores médias e grandes nas áreas estudadas. Essa estrutura diamétrica desbalanceada na sua distribuição, requer acompanhamento ao longo dos próximos anos.

A continuidade das espécies de Caryocar Cuneatum só será possível se houver um acompanhamento 
contínuo da área estudada e se seu manejo for feito de forma sustentável. Onde os seus recursos (sejam econômicos, ambiental e social) possam ser utilizados respeitando todo o ecossistema e entendendo que a floresta precisa sobreviver para garantia de equilíbrio da vida na terra. Essa premissa torna-se válida para todas as espécies de flora do Cerrado. Esse manejo sustentável, torna evidente o desafio principal de sustentabilidade de florestas: explorar sem comprometer a rica biodiversidade do Bioma.

\section{REFERÊNCIAS}

ARAÚJO, F. S.; MARTINS, S. V.; MEIRA NETO, J. A. A.; LANI, J L.; PIRES, I. E.. Estrutura da Vegetação Arbustivo-Arbórea Colonizadora de uma Área Degradada por Mineração de Caulim, Brás Pires, MG. Revista Árvore, v.30, n.1, p.107-116, 2006. DOI: https://doi.org/10.1590/S0100$\underline{67622006000100013}$

ARÉVALO-PINEDO, A.; MACIEL, V. B. V.; CARVALHO, K. M.; COELHO, A. F. S.; GIRALDO-ZUÑIGA, A. D.; ARÉVALO, Z. D. S.; ALVIM, T. C.. Processamento e estudo da estabilidade de pasta de pequi (Caryocar brasiliense). Ciência e Tecnologia de Alimentos, v.30, n.3, p.664-668, 2010. DOI: https://doi.org/10.1590/S0101-20612010000300015

AUSTREgÉSILO, S L.; FERREIRA, R. L C.; SILVA, J. A. A.; SOUZA, A L.; MEUNIER, I. M. J.. Comparação de métodos de prognose da estrutura diamétrica de uma floresta estacional semidecidual secundária. Revista Árvore, v.28, n.2, p.227232, 2004. DOI: https://doi.org/10.1590/S0100$\underline{67622004000200009}$

BARROSO, A. G.; SANO, E. E.; FREITAS, D. M.. Identificação de Desmatamentos Recentes no Cerrado Utilizando as Técnicas de Diferença de Imagens e Índice de Queimada por Diferença Normalizada. Revista Brasileira de Cartografia, v.67, n.7, p1397-1409, 2019.

BATISTA, A. P. B.; APARICÍO, W. C. S.; APARÍCIO, P. S.; SANTOS, V. S.; LIMA, R. B.; MELLO, J. M.. Caracterização estrutural em uma floresta de terra firme no estado do Amapá, Brasil. Pesq. Flor. Bras., v.35, n.81, p.21- 33, 2015. DOI: https://doi.org/10.4336/2015.pfb.35.81.689

BRANNSTROM, C.; JEPSON, W.; FILIPPI, A. M.; REDO, D.; XU, Z.; GANESH, S.. Land change in the Brazilian Savanna (Cerrado), 1986-2002: comparative analysis and implications for land-use policy. Land Use Policy, v.25, n.4, p.579-595, 2009. DOI:

https://doi.org/10.1016/j.landusepol.2007.11.008Get

BRANNSTROM, C.. South America's Neoliberal Agricultural Frontiers: Places of Environmental Sacrifice or Conservation Opportunity?. Ambio: a Journal of the Human Environment, v.38, n.3, p.141-149, 2009.

BÜHLER, E. A.; OLIVEIRA, V. L.. A Neoliberalização da Natureza na Fronteira Agrícola do Cerrado Nordestino. Revista Raízes, Campina Grade, v.39, n.2, p.362-388, 2019. DOI: https://doi.org/10.37370/raizes.2019.v39.110

CORAIOLA, M.; PÉLLICO NETTO, S.. Análise da estrutura dimensional de uma floresta estacional semidecidual localizada no município de Cássia/MG: estrutura volumétrica. Revista Acadêmica. Ciências Agrárias e Ambientais, v.1, n.4, p.11-24, 2003.
DOI: $\underline{\text { http://dx.doi.org/10.7213/cienciaanimal.v1i4.14961 }}$

CONTINI, E.; GASQUES, J. G.; ALVES, E.; BASTOS, E. T.. Dinamismo da agricultura brasileira. Revista de política agrícola, v.19, p.42-64, 2010.

DUARTE, J.; RODRIGUES, N.. Mapeamento inédito mostra uso e cobertura do Cerrado. Embrapa, 2015.

FELFILI, J. M.. Diameter and height distributions in a gallery forest community and some of its main species in central Brazil over a six-year period (1985-1991). Revista Brasileira de Botânica, v.20, p.155-162, 1997. DOI: https://doi.org/10.1590/S0100-84041997000200006

FERREIRA, G. C.. Diretrizes para coleta, herborização e identificação de material botânico nas Parcelas Permanentes em florestas naturais da Amazônia brasileira. Manaus: GT Monitoramento de Florestas, 2006.

GONÇALVES, H.; MENEGuZzO, I. S.; MORO, R. S.. Políticas públicas para a conservação do Bioma Cerrado no Estado do Paraná, Brasil. Terra Plural, v.13, n.1, p.138-152, 2019. DOI: http://dx.doi.org/10.5212/TerraPlural.v.13i1.0009

HIGUCHI, N.; SANTOS, J.; LIMA, A. J. N.. Biometria Florestal. IMF, 2008.

IBGE. Instituto Brasileiro de Geografia e Estatística. Cidades. Rio de Janeiro: IBGE, 2016.

IBGE. Instituto Brasileiro de Geografia e Estatística. Manual Técnico da Vegetação Brasileira. Rio de Janeiro: IBGE, 2012.

LIMA, J. P. C.; LEÃO, J. R. A.. Dinâmica de Crescimento e Distribuição Diamétrica de Fragmentos de Florestas Nativa e Plantada na Amazônia Sul Ocidental. Floresta e Ambiente, v.20, n.1, p.70-79, 2013. DOI: https://doi.org/10.4322/floram.2012.065

MACHADO, F. S.. Manejo de produtos florestais não madeireiros: um manual com sugestões para o manejo participativo em comunidades da Amazônia. PESACRE, 2008.

MACHADO, S. A.; BARTOSZECK, A. C. P.; FIGUEIREDO, A.; OLIVEIRA, E. B.. Dinâmica da distribuição diamétrica de Bracatingais na região metropolitana de Curitiba. Revista Árvore, v.30, n.5, 2006. Dol: https://doi.org/10.1590/S010067622006000500009

MDA. Sistema de informação Territorial. Bacia do Rio Grande/BA: desenvolvimento territorial. Caderno Territorial, 2011.

MENKE, A. B.; CARVALHO JUNIOR, O. A.; GOMES, R. A. T.; 
MARTINS, E. S.; OLIVEIRA, S. N.. Análise das mudanças do uso agrícola da terra a partir de dados de sensoriamento remoto multitemporal no município de Luís Eduardo Magalhães (BA - Brasil). Sociedade \& Natureza, v.3, p.315326, 2009. DOI: https://doi.org/10.1590/S1982$\underline{45132009000300007}$

MIRANDA, E. E.; CARVALHO, C. A.. Na Agricultura, a Preservação dos Cerrados. Revista AgroDBO, p.1-4, 2015.

MMA. Ministério do Meio Ambiente. Plano de Ação para Prevenção e Controle do Desmatamento e das Queimadas no Cerrado. MMA, 2009.

NETTO, C. C.. Dinâmica da Distribuição Diamétrica de Povoamentos de Pinus Taeda L. em Diferentes Idades e Espaçamentos. Dissertação (Mestrado em Engenharia Florestal) - Universidade Federal de Santa Maria, Santa Maria, 2008.

OLIVEIRA, M. E. B.; GUERRA, N. B.; BARROS, L. M.; ALVES, R. E.. Aspectos agronômicos e de qualidade do pequi. Embrapa Agroindústria Tropical, 2008.

OLIVEIRA, M. E. B.; GUERRA, N. B.; MAIA, A. H. N.; ALVES, R. E.; MATOS, N. M. S.; SAMPAIO, F. G. M.; LOPES, M. M. T.. Características químicas e físico-químicas de pequis da Chapada do Araripe, Ceará. Revista Brasileira de Fruticultura, v.32, n.1, p.114-125, 2010. DOI: https://doi.org/10.1590/S0100-29452010005000030

ORELLANA, E.; FIGUEIREDO FILHO, A.; PÉLLICO NETTO, S.; DIAS, A. N.. Modelagem da distribuição diamétrica de espécies florestais em um fragmento de floresta ombrófila mista. Revista Árvore, v.38, n.2, p.297-308, 2014. DOI: https://doi.org/10.1590/S0100-67622014000200010

PEREIRA, L. A.; PINTO SOBRINHO, F. A.; COSTA NETO, S. V.. Florística e estrutura de uma mata de terra firme na Reserva de Desenvolvimento Sustentável Rio Iratapuru, Amapá, Amazônia Oriental, Brasil. Floresta, v.41, n.1, 2011. DOI: http://dx.doi.org/10.5380/rf.v41i1.21191

PRANCE, G. T.; SILVA, M. F.. Caryocaraceae. Flora Neotropica Monograph, v.12, p.1-75, 1973.

ROESLER, R.; MALTA, L. G.; CARRASCO, L. C.; HOLANDA, R. B.; SOUSA, C. A. S.; PASTORE, G. M.. Atividade antioxidante de frutas do cerrado. Ciência e Tecnologia de Alimentos, v.27, n.1, p.53-60, 2007. DOI:

https://doi.org/10.1590/S0101-20612007000100010

SANO, E. E.; PINHATI, F. S. C.. Espaço rural do oeste baiano: identificação de áreas agrícolas sob sistema de plantio direto por meio de dados obtidos por câmera digital e satélite CBERS-2 CCD. Geografia, v.34, n.1, p.117-129, 2009.

SANQUETTA, C. R.. Dinâmica de um remanescente de Floresta Ombrófila Mista no sul do Paraná sob influência de taquaras. Revista Ambiência, v.3, n.1, p.65-78. 2007.
SANTANA, J. A. S.. Padrão de Distribuição e Estrutura Diamétrica de Croton sonderianus Muell. Arg. (Marmeleiro) na Caatinga da Estação Ecológica do Seridó. Revista Verde, v.4, n.3, p.85-90, 2009.

SANTOS, R. O.; ABREU, J. C.; LIMA, R. B.; APARÍCIO, P. S.; SOTTA, E. D.; LIMA, R. C.. Distribuição diamétrica de uma comunidade arbórea na Floresta Estadual do Amapá, Brasil. Biota Amazônia, v.6, n.2, p.24-31, 2015. DOI: http://dx.doi.org/10.18561/21795746/biotaamazonia.v6n2p24-31

SANTOS, W. J.; JACOBSON, T. K. B.; ROCHA.; DINIZ, D. M. S.; SÁ, J. D. A.; BARBOSA-SILVA, D.. Levantamento de espécies lenhosas frutíferas de um cerrado sentido restrito com potencial de exploração, no Assentamento Colônia I, Padre Bernardo/GO. Cadernos de Agroecologia, v.9, n.3, 2014

SCHNEIDER, P. R.; SCHNEIDER, P. S. P.; SOUZA, C. A. M.. Análise de Regressão aplicada à Engenharia Florestal. FACOS, v.2, 2009.

SCHNEIDER, P. R.; FINGER, C. A. G.. Manejo sustentado de florestas inequiânias heterogêneas. Santa Maria: UFSM, 2000.

SEI. Superintendência de Estudo Econômico e Social da Bahia. Perfil Socioeconômico do Município de Baianópolis. Salvador: SEI, 2011.

SILVA, M. R.; LACERDA, D. B. C. L.; SANTOS, G. G.; MARTINS, D. M. O.. Caracterização química de frutos nativos do cerrado. Ciência Rural, v.38, n.6, p.1.790-1.793, 2008. DOI: https://doi.org/10.1590/S0103-84782008000600051

SOUZA, A. L.; JESUS, R. M.. Distribuição diamétrica de espécies arbóreas da Floresta Atlântica: análise de agrupamento. Serviço do Instituto de Florestas, v.1, n.10, p.30, 1994.

SPIEGEL, M. R.. Estatística. Pearson Makron Books, v.3, p.551, 2006.

STRASSBURG, B. N.. Moment of truth for the Cerrado. Nature Ecology \& Evolution, v.1, n.3, 2017.

THIBAU, C. E.. Produção sustentada em florestas: conceitos e tecnologias, biomassa energética, pesquisas e constatações. Gráfica, 2000.

VACCHIANO, M. C.; SANTOS, J. W. C. M.; ANGEOLETTO, F.; SILVA, N. M.. Data Support Claims That Brazil Leads the World in Environmental Preservation?. Environmental Conservation, v.46, n.2, p.118-120, 2019. DOI: https://doi.org/10.1017/S0376892918000371

WWF. World Wildlife Found. The Growth of Soy: Impacts and Solutions. Gland, 2014.

A CBPC - Companhia Brasileira de Produção Científica (CNPJ: 11.221.422/0001-03) detém os direitos materiais desta publicação. Os direitos referem-se à publicação do trabalho em qualquer parte do mundo, incluindo os direitos às renovações, expansões e disseminações da contribuição, bem como outros direitos subsidiários. Todos os trabalhos publicados eletronicamente poderão posteriormente ser publicados em coletâneas impressas sob coordenação da Sustenere Publishing, da Companhia Brasileira de Produção Científica e seus parceiros autorizados. Os (as) autores (as) preservam os direitos autorais, mas não têm permissão para a publicação da contribuição em outro meio, impresso ou digital, em português ou em tradução. 